\title{
A molecular, phylogenetic and functional study of the dADAR mRNA truncated isoform during Drosophila embryonic development reveals an editing-independent function
}

\author{
Sushmita Ghosh, Yaqi Wang, John A. Cook, Lea Chhiba, Jack C. Vaughn* \\ ${ }^{1}$ Department of Biology, Cell Molecular and Structural Biology Program, Miami University, Oxford, USA; \\ *Corresponding Author: vaughnjc@MiamiOH.edu
}

Received 16 August 2013; revised 25 September 2013; accepted 7 October 2013

Copyright (C) 2013 Sushmita Ghosh et al. This is an open access article distributed under the Creative Commons Attribution License, which permits unrestricted use, distribution, and reproduction in any medium, provided the original work is properly cited.

\begin{abstract}
Adenosine Deaminases Acting on RNA (ADARs) have been studied in many animal phyla, where they have been shown to deaminate specific adenosines into inosines in duplex mRNA regions. In Drosophila, two isoform classes are encoded, designated full-length (contains the editase domain) and truncated (lacks this domain). Much is known about the full-length isoform, which plays a major role in regulating functions of voltage-gated ion channel proteins in the adult brain. In contrast, almost nothing is known about the functional significance of the truncated isoform. In situ hybridization shows that both isoform mRNA classes are maternally derived and transcripts for both localize primarily to the developing central nervous system. Quantitative RT-PCR shows that about 35\% of all dADAR mRNA transcripts belong to the truncated class in embryos. 3'-RACE results show that abundance of the truncated isoform class is developmentally regulated, with a longer transcript appearing after the mid-blastula transition. 3'-UTR sequences for the truncated isoform have been determined from diverse Drosophila species and important regulatory regions including stop codons have been mapped. Western analysis shows that both mRNA isoform classes are translated into protein during embryonic development, as full-length variant levels gradually diminish. The truncated protein isoform is present in every Drosophila species studied, extending over a period spanning about $40 \times 10^{6}$ years, implying a conserved function.
\end{abstract}

Previous work has shown that a dADAR protein isoform binds to the evolutionarily conserved rnp-4f pre-mRNA stem-loop located in the 5'UTR to regulate splicing, while no RNA editing was observed, suggesting the hypothesis that it is the non-catalytic truncated isoform which regulates splicing. To test this hypothesis, we have utilized RNAi technology, the results of which support the hypothesis. These results demonstrate a novel, non-catalytic function for the truncated $d A D A R$ protein isoform in Drosophila embryonic development, which is very likely evolutionarily conserved.

Keywords: $d A D A R$ Gene; Truncated $d A D A R$ Isoform; RNAi Knockdown; 5'-UTR Intron Retention; rnp-4f Gene

\section{INTRODUCTION}

The strength of Drosophila as a model organism comes from its amenability for physiological, cellular, molecular and genetic studies. Drosophila has been used as a model genetic organism for over a century. The development of a targeting gene expression approach, capable of driving the expression of any gene in any tissue in a temporal and spatial manner, has proven to be one of the most powerful techniques for study of Drosophila gene function in vivo [1,2]. The development of a genome-wide transgenic RNAi library for protein coding gene inactivation in Drosophila has been invaluable [3]. Completion and annotation of the D. melanogaster genome [4] followed by the sequencing of eleven other Drosophila species' genomes [5] have revealed remarkable similarities to genes involved in human physiologi- 
cal processes and disease. About $60 \%$ of human genes have been found to be orthologous to Drosophila genes, and further analysis of these genes with their alternatively spliced transcripts will surely be crucial for better understanding the nature of human disease [6]. Among human cancer genes alone, $68 \%$ have orthologs in Drosophila. The $d A D A R$ and $r n p-4 f$ genes which are the central focuses in this paper are both represented by human orthologs.

Adenosine Deaminases Acting on RNA (ADARs) have been described from throughout the animal kingdom, where they function to co-transcriptionally deaminate specific (or non-specific) adenosine residues within pre-mRNAs [reviewed in 7,8] or pre-miRNAs [9]. The first example of RNA editing in Drosophila was described in our lab [10,11] within some adult brain rnp-4f mRNA transcripts. In Drosophila the single ADAR gene, designated $A A D A R$ [12], can produce several different full-length mRNA isoforms by alternative splicing, which contain a catalytic deaminase domain. Differential 3 '-end formation arising by termination within intron 6 produces truncated transcripts lacking the deaminase domain [13]. Among the best-studied examples of nucleotide-specific deamination by ADARs are mRNAs encoding mammalian [reviewed in 14] and several different Drosophila brain ligand- or voltage-gated ion channel proteins [reviewed in 15]. In situ localization studies have shown that $d A D A R$ mRNAs are primarily located in the ventral nerve cord and brain of the developing embryo $[12,16]$. There is a growing body of evidence that ADARs also have various editing-independent functions, likely arising from their roles as RNA-binding proteins [reviewed in 15]. In contrast to what is known about catalytically active full-length $d A D A R$ s, virtually nothing is known about the functions or phylogenetic distribution of the truncated $A A D A R$ isoform.

RNP-4F, which is encoded by the Drosophila nuclear gene $r n p-4 f[11,17]$, is believed to function as a spliceosome assembly factor. Studies on its homologues human p110/SART3 and yeast Prp24 have shown that RNP-4F changes the U6-snRNP secondary structure and promotes base-pairing to U4-snRNA during spliceosome assembly [18,19]. In Drosophila, developmental Northerns [20] and RT-PCR analysis [21] have shown that there are two major $r n p-4 f$ mRNA isoforms during fly development, which have been designated "long" and "short," differing by an alternatively spliced 177-nt sequence located in the 5'-UTR region. These studies have also shown that during embryo development, the abundances of the two $r n p-4 f$ isoforms are developmentally regulated. In situ localization studies have shown that the 5'-UTR unspliced mRNA isoform is primarily located in the ventral nerve cord and brain of the developing embryo [21]. Computer-predicted RNA folding has sug- gested that the 177-nt long $r n p-4 f$ isoform-specific sequence located in the 5'-UTR can form an evolutionarily-conserved stem-loop due to intron 0 pairing with part of adjacent exon 2 [16]. We have recently combined RNA electrophoretic mobility shift and mutational analysis to show that a Drosophila $d A D A R$ protein isoform binds to the $r n p-4 f$ pre-mRNA stem-loop and regulates alternative splicing [22]. However, it is not yet known which isoform has this function. Here, we report results of a study on the molecular, phylogenetic and functional characterization of the Drosophila $d A D A R$ truncated isoform during embryonic development, using a variety of techniques including RNAi technology.

\section{MATERIALS AND METHODS}

\subsection{Fly Stocks and Embryo Preparation}

Drosophila melanogaster red eye wild-type strain Oregon R and white eye mutant $\mathrm{w}^{1118}$ were obtained from the Bloomington, IN Stock Center. Additional species were obtained from the Drosophila Species Stock Center, San Diego, CA. Fly propagation and embryo collection methods for materials to be processed for RNA or protein isolations or for in situ hybridizations were as previously described [21,23]. Embryo staging was as described [24]. Abbreviations for fly species used in this study are: D. mel (D. melanogaster); D. sim (D. simulans); D. sec (D. sechellia), D. mau (D. mauritiana); D. yak (D. yakuba); D. ere (D. erecta); D. ana (D. ananassae) and D. ame (D. americana).

\subsection{Localization of $d A D A R$ mRNA Isoforms by in Situ Hybridization}

Genomic DNA fragments specific to combined fulllength and truncated $d A D A R$ isoforms within exon-4a (primer set "A"), to specifically the full-length isoform within exon 7 (primer set "B"), and specifically to the truncated isoform terminating within intron 6 (primer set "C") were obtained by PCR, based on the known $d A D A R$ gene sequence [12]. The forward PCR primer for set " $A$ " was 5'-CTAATGCAATGTAATGCAGC-3' and the reverse sequence was 5'-CCTCCTCGCTTCGCTGTT-3'. The forward PCR primer sequence for set "B" was 5'-CCACAGCATATCAGTCGATT-3' and the reverse sequence was 5'-CTATTTGGATGTTTATCAACAC-3'. The forward PCR sequence for set "C" was 5'-CCATAGAACTTAAATCTAAGAG-3' and the reverse sequence was 5'-CCTTCTAAAACGATCAGACG-3'. These DNA fragments were ligated into "pGEM-T Easy" plasmid expression vector (Promega), and orientation of fragment inserts was verified by DNA sequencing. In vitro transcriptions of sense (control) and antisense (complementtary to mRNA transcripts) RNA probes labeled with digoxigenin-11-dUTP (DIG) were carried out using a com- 
mercially available kit (Promega), followed by hybridizations as described [16,25]. Locations of nuclei in early embryos were determined by DAPI staining.

\subsection{Total Cell RNA and Protein Isolations from Fly Embryos}

Total cell RNAs were isolated from about 50 ug of tissue using the Maxwell 16 IVD instrument following the directions of the supplier (Promega) and stored at $-80^{\circ} \mathrm{C}$ until used. Proteins were isolated from $100 \mathrm{mg}$ of embryos, as described [16], and also stored at $-80^{\circ} \mathrm{C}$. RNA and protein concentrations were estimated by $\mathrm{OD}_{260}$ and $\mathrm{OD}_{280}$ spectrophotometry, respectively (NanoDrop Technologies).

\subsection{Semi-Quantitative Reverse Transcription-Polymerase Chain Reaction (RT-PCR) for dADAR mRNA Isoform Relative Abundance}

Reverse transcription of total cell RNAs isolated from pooled 0 - $18 \mathrm{~h}$ stage embryos and the associated negative controls were carried out as previously described $[16,23]$. A PCR primer set "In-6" was designed to detect specifically the truncated $d A D A R$ mRNA isoform, where the forward primer sequence located in exon 6 was 5'-GCGGTGAGCATATGAGTG-3' and the reverse primer sequence located in intron 6 upstream of the shortest 3'-end detected by 3'-RACE was 5'-GTAAACTGAACTTAGCATATAC-3', predicted to amplify a 155 bp fragment. A second PCR primer set "Ex-7" was designed to specifically amplify the full-length $d A D A R$ mRNA isoform to amplify a 168-bp fragment, using the forward and reverse primer sequences described in section 2.2 for "primer set B". PCR cycle numbers in the linear range wherein band intensities following gel electrophoresis were shown to be proportional to quantity of cDNA product were determined for each primer set. Gels were stained with SYBR Green I (Invitrogen) and scanned in a Molecular Dynamics Storm 860 phosphorimager, using the blue fluorescence mode. Band intensity quantifications were obtained using version 5.2 of the Image Quant software. Results were expressed as percentage of each isoform compared to the combined band intensities.

\subsection{Developmental and Phylogenetic 3'-RACE of Truncated dADAR mRNA Isoforms}

The 3'-termini of truncated $d A D A R$ mRNA isoforms within intron 6 during $D$. melanogaster embryo development, first larval instar, and in adult male and female flies were precisely determined by 3'-RACE using a commercially available kit and following the directions of the supplier (Ambion). The 3'-termini of related species was carried out on pooled $0-18 \mathrm{~h}$ stage embryos. The sequence of the outer gene-specific PCR primer, located in highly conserved exon 6, was 5'-GCGTTGTCTT CTCAAATATTTAT-3' and that of the nested inner PCR primer also located in exon 6 was 5'-GCACAGCTGG ACCTTCAGT-3'. These primers were effective for species nearer to the top of the phylogenetic tree, but due to sequence divergence the corresponding primers for $D$. ananassae were 5'-GCGGTGTCTCCTCAAGTACTTAT3 ' and 5'-GCCCAGCTGGATCTTCAGT-3', and for $D$. americana were 5'-CGCTGTCTTCTAAAATACTTGT3' and 5'-ATGCTCAACTTGATCTCCAGT-3'. Electrophoresis in $2 \%$ agarose gels containing ethidium bromide was followed by cDNA band excision and fragment purification using the "QIAquick" gel extraction kit (Qiagen). Sequencing was done on an ABI 3100 automated sequencer and data interpreted using EditView software. The precise 3'-terminal nucleotides were identified with reference to genomic DNA sequences, facilitated by location of the poly(A)-tail.

\subsection{Developmental and Phylogenetic Western Immunoblot Analysis of dADAR Protein Isoforms}

Expression of $d A D A R$ mRNAs into protein from specifically full-length or truncated isoforms was determined using Western immunoblotting. In D. melanogaster, protein extract from embryo developmental stages was used. In related species, protein extract from pooled 0 $18 \mathrm{~h}$ stage embryos was utilized. A polyclonal affinity-purified antibody directed against the highly conserved $D A D A R$ protein amino acid sequence -RGYEM PKYSDPKKKC-encoded primarily by nucleotides located at the 3'-end of exon 1 [12] was commercially produced (GenScript). This antibody would be expected to recognize both full-length and truncated isoforms in a wide variety of Drosophila species, the isoform classes being recognizable based on their differing sizes [16]. Western immunoblotting was carried out as previously described [16], with modifications including electrophoresis of about 100 ug embryo protein extract in precast $4 \%$ - $15 \%$ gradient SDS-polyacrylamide mini-PROTEAN TGX gels (BioRad) and protein transfer to $0.45 \mathrm{um}$ nitrocellulose membranes. The stock $2.2 \mathrm{mg} / \mathrm{ml}$ primary antibody produced in rabbit was diluted 1:1,000 and after incubation/washing membranes were incubated in donkey anti-rabbit HRP-conjugated secondary antibody with stock concentration $1 \mathrm{mg} / \mathrm{ml}$ (Abcam no. ab97064) diluted 1:5000. As loading controls, membranes were probed with mouse anti- $\alpha$-tubulin antibody (Developmental Studies Hybridoma Bank no. AA4.3) diluted 1:2000, followed by incubation in rabbit anti-mouse HRP-conjugated secondary antibody with stock concentration 1.5 
$\mathrm{mg} / \mathrm{ml}$ (Invitrogen) diluted 1:5000. Negative controls were run using secondary antibody in the absence of prior primary antibody. Signals were detected using the ECL detection kit (GE Healthcare) and Blue Basic Autorad film (GeneMate). Band intensities were quantified after scanning films into the computer and employing Image Quant software. Results were expressed as ratio intensity of $d A D A R$ isoform to that of $\alpha$-tubulin.

\subsection{Influence of Specific $d A D A R$ Protein Isoforms on 5'-UTR Intron Splicing Regulation in rnp-4f Pre-mRNA Using RNAi Technology}

To test the hypothesis that it is the truncated $d A D A R$ protein isoform which is utilized during development to bind the 5'-UTR stem-loop within $r n p-4 f$ pre-mRNA and inhibit splicing [16,21-22] we utilized RNAi technology. A UAS-driven D. melanogaster RNAi transgenic fly line (stock \#7763) containing an inverted repeat directed against $d A D A R$ exon 9 and inserted into chromosome 3 was obtained from the Vienna Drosophila RNAi Center (VDRC). This would be expected to specifically knock down full-length $d A D A R$ transcripts, since the truncated isoform does not contain exon $9[13,16]$. To express the RNAi insert [1,2], virgin females containing the RNAi insert were crossed with males from a GAL4 fly line (stock \#458) containing the tissue specific expression promoter elav-Gal4, obtained from the Bloomington, IN Stock Center, which directs expression to the developing central nervous system by around $8 \mathrm{~h}$ of embryo development. Embryos for this work were therefore collected from the $8-16 \mathrm{~h}$ stage. The expected activity of this RNAi construct on specifically the full-length $d A D A R$ isoform following RNAi expression was tested by semiquantitative RT-PCR to compare the results to $\mathrm{w}^{1118}$ flies in which the RNAi insertion had been made, as described above in section 2.4. We then examined the influence of specifically knocking down the full-length dADAR protein isoform on splicing of the 5'-UTR intron in $r n p-4 f$ pre-mRNA, in comparison with $\mathrm{w}^{1118}$, using semi-quantitative RT-PCR. This was done by bracketing the rnp-4f 5'-UTR intron 0 site with a primer set having the forward sequence 5'-ATTCGCATATTATTCACACT-3' and reverse sequence 5'-GATCAGATCATACTCGTC-3' [20].

\section{RESULTS AND DISCUSSION}

\subsection{Localizations of Specific dADAR mRNA Isoforms During Embryo Development in D. melanogaster}

The in situ hybridization results (Figures 1(a)-(c)) clearly show that neither $D A D A R$ mRNA isoform is de- tectable in the cytoplasm of embryos at the cellular blastoderm stage, $2-3 \mathrm{~h}$ after fertilization. In contrast, heavy localization is seen in the yolk region for both isoforms. These results are interpreted to show that both isoforms are maternally derived, and that little if any transcription is occurring at this early stage. The locations of DAPIstained nuclei (Figure 1(d)) in comparison to the diagrammatic sketches (Figures 1(e) and (f)) help to clarify what is happening here. The developing ventral nerve cord and brain in mid-stage embryos (Figures 1(g)-(j)) show identical localizations for both $d A D A R$ mRNA isoforms, with strong signal in both regions, as well as in the yolky region of the gut. The signal in yolk is probably residual from that present in very early embryos, while that in the developing central nervous system is likely newly transcribed.

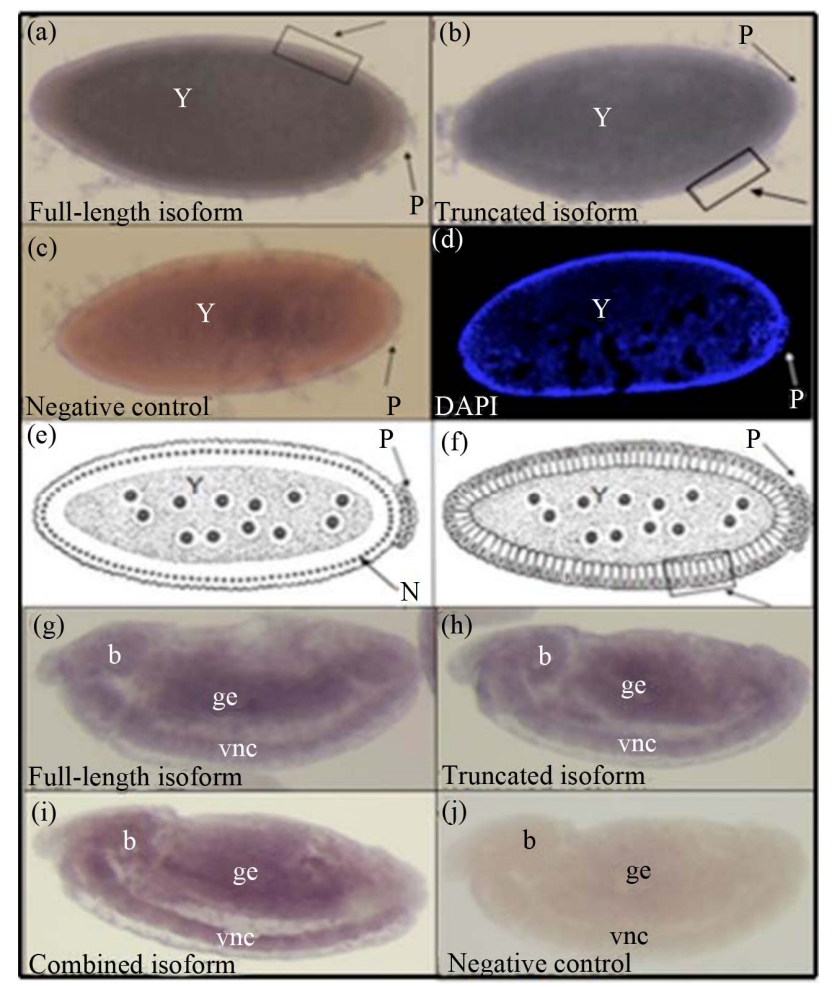

Figure 1. DIG-labeled RNA probe in situ hybridization for specific $d A D A R$ mRNA isoforms in D. melanogaster. Cellular blastoderm stage (2-3 $\mathrm{h}$ after fertilization) localization patterns are shown in A-C. DAPI staining shows nuclear positions at the embryo perimeter (d). Diagrammatic sketches [26] depict developmental changes between the syncytial blastoderm stage (e) with nuclei at the perimeter and absence of cell membranes in the cellular blastoderm stage (f). Localization of $d A D A R$ mRNAs is restricted to the central yolk region (Y), being absent from the peripheral cell cytoplasm (boxed) and pole cells (P). By stage $14(\sim 11 \mathrm{~h}$ after fertilization), both $d A D A R$ isoforms localize $(\mathrm{g}-\mathrm{j})$ to the developing ventral nerve cord (vnc) and brain (b), as well as in the yolky gut region (ge). Embryo views are lateral with anterior to the left and ventral side down. 


\subsection{IADAR mRNA Studies}

Developmental 3'-RACE (Figure 2) for truncated dADAR mRNA shows that prior to the mid-blastula transition (MBT) stage, which occurs at about $3 \mathrm{~h}$ after fertilization, a single truncated isoform band is present. Subsequently, a switch occurs and a second band appears, which is of greater length than the first and persists throughout the embryo stages, but is absent from $1^{\text {st }}$ instar as well as adult males and females. The authenticity of these gel bands was verified by excision and sequencing. It is significant that the longer isoform appears when the central nervous system is developing and persists as that process continues. This isoform appears to be newly transcribed following the MBT and is probably the transcript observed during the in situ hybridizations within the developing central nervous system (Figures 1(g)-(i)). In contrast, the shorter isoform is present as early as the 0 - $1 \mathrm{~h}$ stage (not shown) and is probably the maternally derived transcript observed by in situ hybridization. It is clear from these results that the truncated $d A D A R$ mRNA isoform is developmentally regulated and exists in two different lengths. Many Drosophila genes are maternally transcribed and passed on to the fertilized egg, but a large fraction of them are subsequently degraded at the MBT owing to binding of the protein SMG and recruitment of a destabilizing complex [27]. Our results show that this is not the case for either the full-length or truncated $I A D A R$ mRNA transcripts.

To determine whether or not a truncated $D A D A R$ mRNA isoform is present in species related to D. melanogaster, the 3'-RACE work was extended to other species. It was found that every species studied contains two differently sized truncated isoforms. In this work, each gel cDNA band (not shown) was excised and sequenced, followed by their comparison in a nucleotide alignment (Figure 3). Every sequence is truncated within intron 6 , contains a potential stop codon located not far from the 5 '-end of

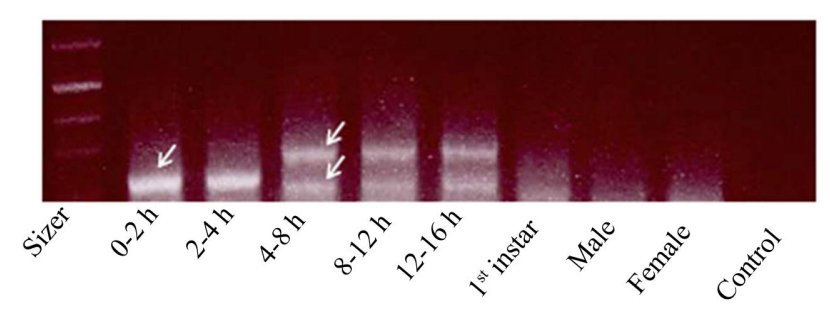

Figure 2. Developmental 3'-RACE of truncated $d A D A R$ mRNA isoform in D. melanogaster. A single shorter isoform (single arrow) is observed prior to the mid-blastula transition, which occurs at about $3 \mathrm{~h}$ after fertilization. The abundance of this shorter isoform then diminishes as a longer isoform (two arrows) is observed in later embryo stages. Both truncated isoform variants are absent in $1^{\text {st }}$ instar as well as in adult male and female flies. All cDNA bands were verified by excision from gels and sequencing.

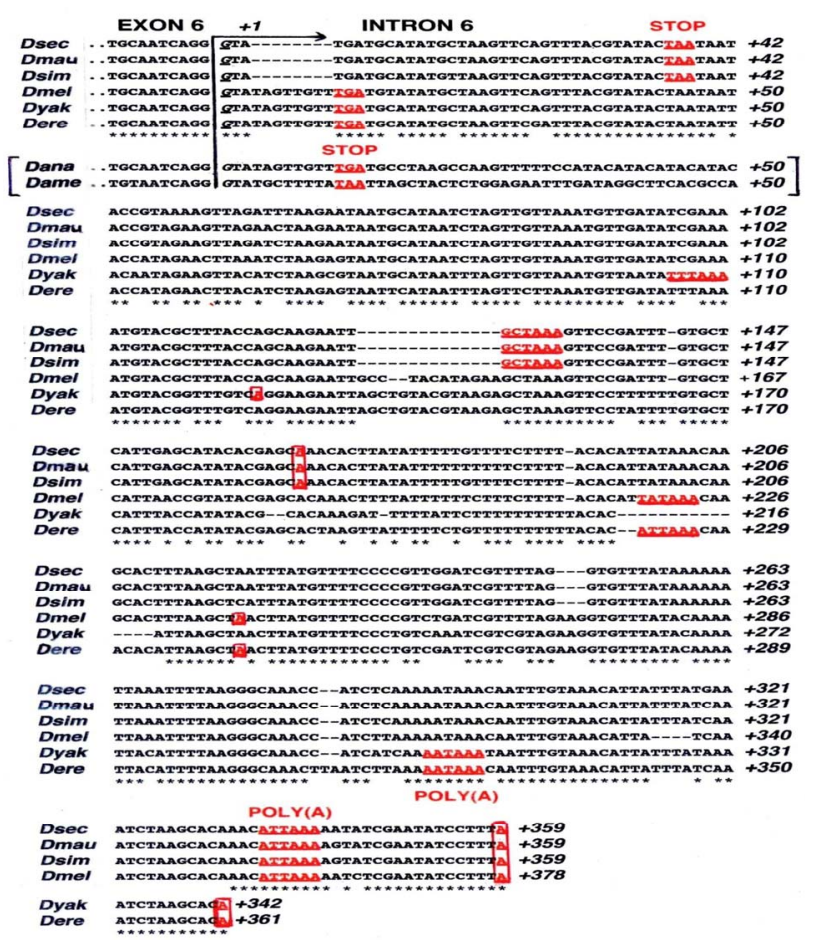

Figure 3. Sequence alignment of experimentally derived truncated $d A D A R$ mRNA isoform 3'-UTR and 3'-termini in diverse Drosophila species as determined by 3'-RACE. Sequences for $D$. ananassae and D. americana were too divergent from the others to enable meaningful comparison, and are only partially shown. Positions of potential regulatory elements including stop codons and poly(A)-signals (underlined) are indicated. Terminal nucleotides immediately prior to the poly(A)-tail are boxed. All species studied contain two different truncated isoform lengths, as shown by the internally boxed nucleotides. Conserved nucleotides are marked by asterisks.

intron 6 , and contains a potential poly(A)-signal located shortly upstream of the sequence-verified poly(A)-tail. The sequences of these probable poly(A)-signals show some variability, which is not surprising, given the known diversity of such signals in other Drosophila mRNAs [28].

Full-length and truncated $D A D A R$ mRNA isoforms are present in all $D$. melanogaster embryo stages, as previously shown using in situ hybridization [16]. The in situ hybridization results reported in this study (Figure 1) show that the two isoforms are located in identical sites within very early as well as mid-stage embryos, but do not permit learning their relative abundance. We determined the relative abundance of the two isoform classes in pooled $0-18 \mathrm{~h}$ stage embryos by semi-quantitative RT-PCR. This was done by using PCR primer sets specific to each isoform class (Figure 4(a)). Following gel electrophoresis and staining with SYBR green I (Figure 4(b)), band intensities were determined. Quantification (Figure 4(c)) shows that the full-length $d A D A R$ mRNA isoform comprises about $65 \%$ of the total gene tran- 
(a)

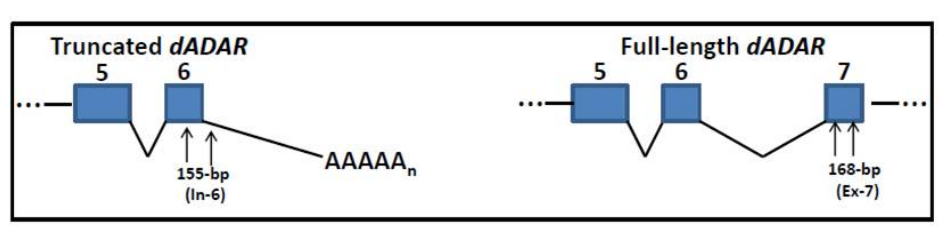

(b)

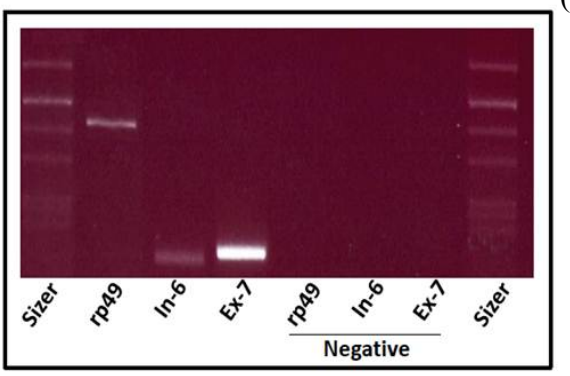

(c)

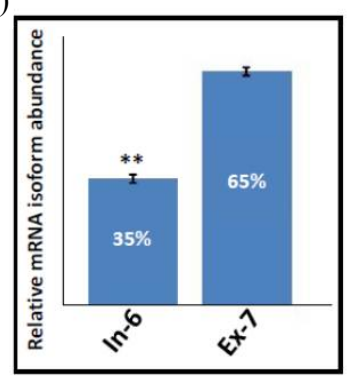

Figure 4. Semi-quantitative RT-PCR for $d A D A R$ mRNA isoform relative abundance in $D$. melanogaster. Orientation diagram (a) shows positions of isoform-specific PCR primers. Gel electrophoresis of PCR products (b) shows relatively high levels of full-length isoform (Ex-7) in comparison to truncated isoform (In-6) in ethidium bromide stained gel. Quantification of band intensities in gels stained with SYBR green I is shown in panel (c). Constitutively expressed $r p-49$ mRNA was included as a reference against which the other band intensities were compared.

scripts, with reference to constitutively-expressed $r p-49$ [29]. The location of the downstream PCR primer for detection of truncated isoforms was upstream of the 3'-end of the shortest isoform (Figure 3), so that both truncated isoform size variants are included in this comparison.

\section{3. $d A D A R$ Protein Studies}

We studied the expression of both full-length and truncated $d A D A R$ mRNAs into protein using Western immunoblotting (Figure 5(a)). The full-length protein isoform is recognized by a band sized at about $70 \mathrm{kDa}$ [12]. It was found that the abundance of full-length $d A D A R$ protein relative to the $\alpha$-tubulin reference is highest at $0-2 \mathrm{~h}$ after fertilization. This protein probably represents a maternal contribution to the fertilized egg, being comparable to that previously described for RNP4F protein using whole embryo tissue immunostaining [21]. It is observed that during embryonic development, the abundance of full-length protein isoform diminishes, with a plateau in mid-embryo stages (Figure 5(c)). The Westerns also show a very faint band sized at about 41 $\mathrm{kDa}$ in all developmental stages, clear evidence that the truncated mRNA is expressed into protein. Preliminary quantification results (Figure 5(d)) show that the truncated protein isoform occurs at a constant $\sim 16 \%$ of the full-length isoform at every developmental stage.

To determine whether or not the truncated $d A D A R$ protein isoform is present in species related to $D$. melanogaster, which if true could be interpreted to show that this isoform performs some conserved function, the Western analysis was extended to include embryos from other species (Figure 6). It was found that in every species studied, the full-length and also truncated $d A D A R$ protein isoforms are present. In common with the results obtained for $D$. melanogaster, the relative abundance of the truncated isoform was found to be very low in related species.

\subsection{RNAi Studies}

Previous work has shown that an unidentified $d A D A R$ protein isoform binds to the 5'-UTR stem-loop of $r n p-4 f$ pre-mRNA to regulate alternative splicing $[16,22]$. To determine the $A A D A R$ protein isoform which regulates splicing in the 5'-UTR control region, we utilized RNAi technology. The 3'-UTR sequence of the D. melanogaster truncated $d A D A R$ isoform (Figure 3) is a simple one, being comprised from part of intron 6, and like many other introns lacks diversity in nucleotides which would be required to construct a transgenic fly line with RNAi activity having the required specificity. We therefore utilized a UAS-driven transgenic RNAi line directed against exon 9 available from the VDRC, expected to knock down the full-length $D A D A R$ mRNA isoform, described in Materials and Methods. We began by verifying that this RNAi transgenic fly line would specifically knock down the full-length $d A D A R$ mRNA isoform. This was done by crossing the UAS-RNAi line with a GAL4 line containing the tissue specific expression promoter elav-Gal4, which directs expression to the central nerv- 
(a)

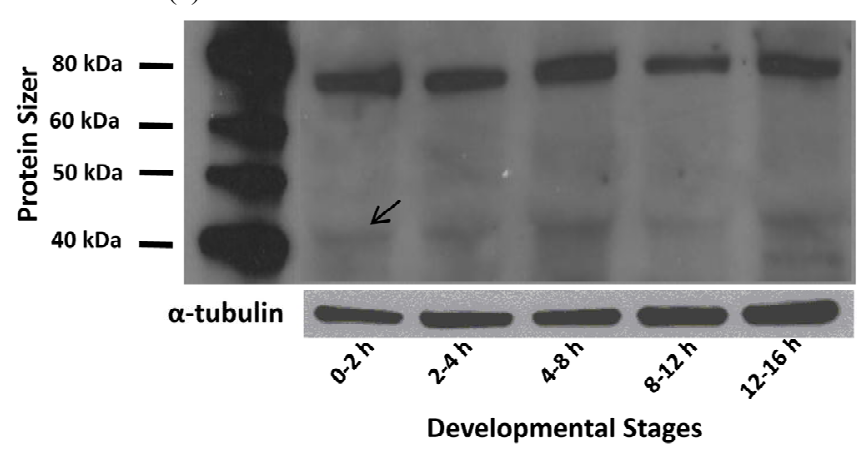

(c)

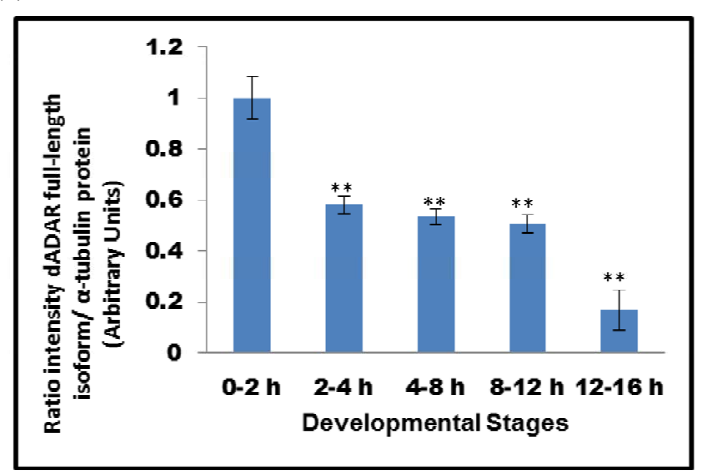

(b)

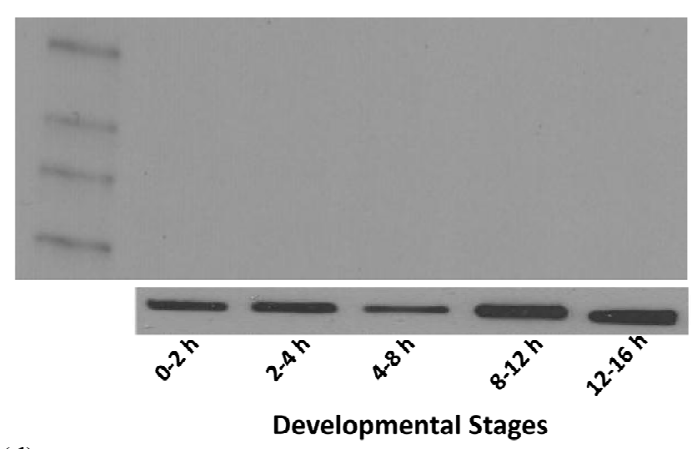

(d)

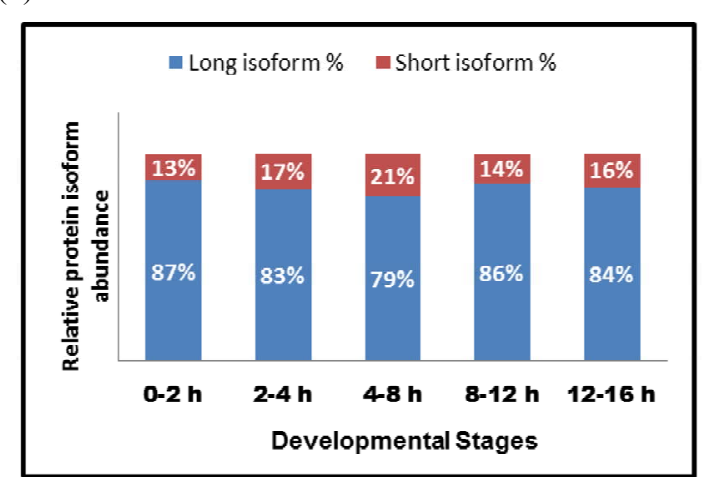

Figure 5. Developmental Western immunoblot analysis of $d A D A R$ protein isoforms in D. melanogaster. (a) Both classes of mRNA isoform are expressed into protein at every developmental stage, as indicated by a prominent band at $\sim 70 \mathrm{kDa}$ for the full-length isoform and a very faint band at $\sim 41 \mathrm{kDa}$ for the truncated isoform (arrow). (b) Negative control. (c) Quantification of full-length protein isoform abundance in comparison to constitutively expressed $\alpha$-tubulin. (d) Relative abundance of full-length and truncated protein isoforms during development.

ous system by around $8 \mathrm{~h}$ of embryo development. Embryos were pooled from the $8-16 \mathrm{~h}$ stage and RNA was extracted. The two PCR primer sets used for estimation of relative $A A D A R$ isoform relative abundance (Figure 4(a)) were employed, and gel electrophoresis was run in comparison to $\mathrm{w}^{1118}$ embryos collected from the same developmental period, since the RNAi line had been constructed in this fly line. The results (Figure 7(b)) show that the full-length $d A D A R$ isoform mRNA is specifically knocked down, as expected. Knockdown is not complete, as is commonly observed in such experiments. We then determined the effect of specifically knocking down the full-length $d A D A R$ isoform upon splicing of the 5'-UTR intron in rnp-4f pre-mRNA, in comparison with $\mathrm{w}^{1118}$ by itself, again using semi-quantitative RT-PCR. This was done by bracketing the rnp-4f 5'-UTR intron 0 site with a primer set whose location is indicated in Figure 7(a). Our reasoning was that if the full-length dADAR protein isoform binds to the stem-loop in wildtype, represented by $\mathrm{w}^{1118}$, to inhibit alternative splicing, then specific removal of this $d A D A R$ protein isoform would result in diminished splicing. However, this result was not obtained, and instead normal splicing is seen (Figure 7(c)). This result is interpreted to show that it is the truncated $A A D A R$ protein isoform which binds to the stem-loop to inhibit splicing.

\section{CONCLUSIONS}

Most eukaryotic translational control elements are located in the 5'- and 3'-UTR of mRNAs, and have been shown to play several different roles in the regulation of gene expression, including translational modulation [reviewed in 30]. The controls of both translational efficiency and mRNA degradation are important aspects in regulation of eukaryotic gene expression [31]. Numerous observations have pointed to a $d A D A R$ protein isoform playing a major direct role during intron splicing regulation at the rnp-4f 5'UTR control region. It was shown early [20] that two different $r n p-4 f$ mRNA isoforms exist during Drosophila development, termed "long" (unspliced) and "short" (alternatively spliced). Developmental Northerns have shown that abundance of these two isoforms is developmentally regulated, the unspliced isoform becoming most abundant in mid-embryo stages when the central nervous system is forming [20,21]. The coding potential for the two isoforms is identical, and they only differ in the presence or absence of a 5'-UTR containing an RNA duplex stem-loop. This observation suggested 
(a)

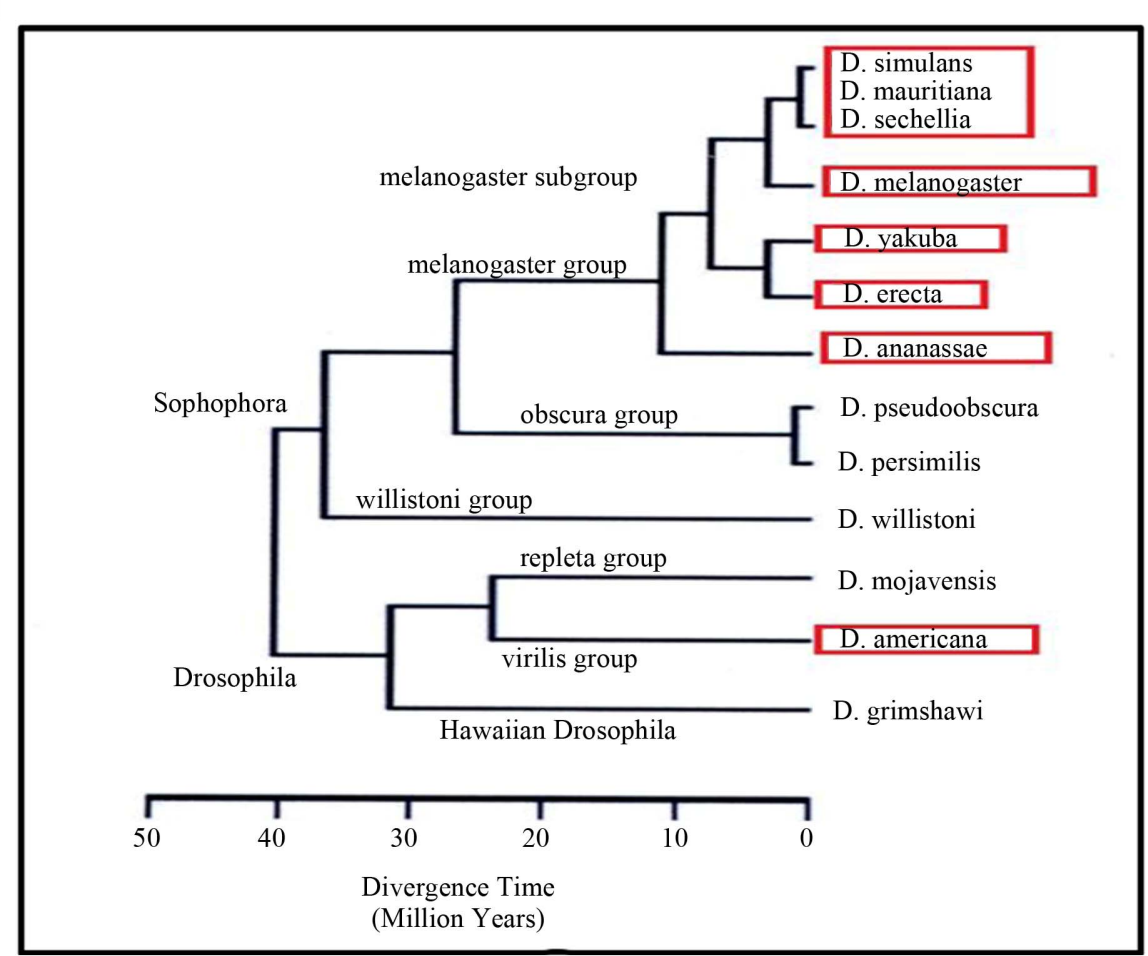

(b)

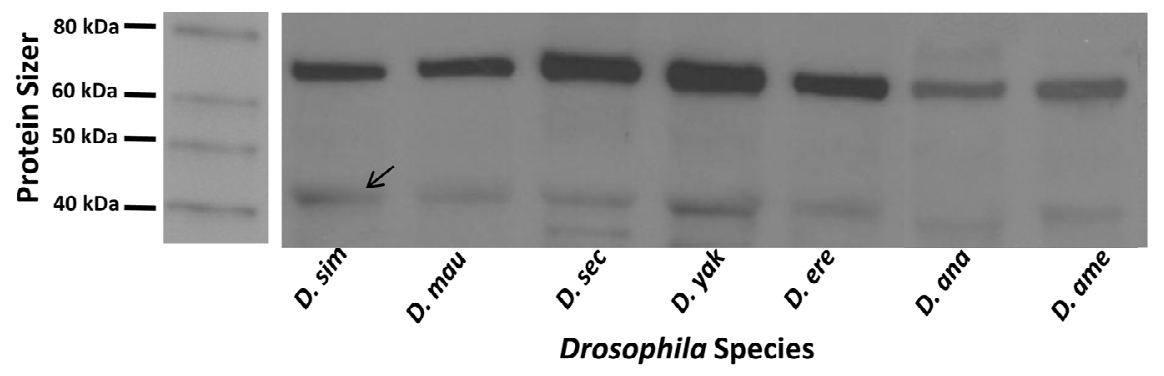

Figure 6. Phylogenetic Western immunoblot analysis of $d A D A R$ protein isoforms in diverse Drosophila species. (a) Phylogenetic tree depicting species (boxed) included in this study [modified from reference 5]. (b) Western blot results, showing that both $d A D A R$ mRNA isoforms are expressed into protein in every species studied, the truncated isoform being only very weakly expressed (arrow).

that the splicing decision depends on some feature within the 5'-UTR. It was found that both $r n p-4 f$ [21] and dADAR mRNA isoforms [16] localize to the developing central nervous system in Drosophila. Insofar as $d A D A R$ has a strong preference to bind duplex RNAs, we began to suspect that this protein may play a role in the splicing decision by binding to the stem-loop, perhaps operating by steric interference. To test this hypothesis, RNA electrophoretic mobility shift analysis utilizing labeled synthetic stem-loop RNA probes showed that band shifts do occur in vitro when protein extract from wild-type embryos is incubated with the probe [22]. However, this effect was not observed when protein extract from a $d A D A R$ null mutant fly line was used. It was also shown in this same study by using real-time qRT-PCR that dur- ing embryogenesis unspliced $r n p-4 f$ levels diminish by up to $85 \%$ in the $d A D A R$ null mutant, interpreted to show that a $d A D A R$ protein isoform plays a direct role in rnp-4f 5'-UTR splicing regulation in vivo. We have proposed a working model to explain the role of a $d A D A R$ protein isoform on $r n p-4 f$ pre-mRNA alternative intron splicing regulation [16]. It has been found using RT-PCR of numerous individually cloned $r n p-4 f$ mRNA stem-loop regions from mid-embryo stages that no RNA editing occurs [16]. This could have been interpreted to show that it is the truncated $D A D A R$ protein isoform which is important in splicing regulation, since this isoform lacks the deaminase editing domain. However, it is well known that for unknown reasons full-length $d A D A R$ isoform protein scarcely exhibits any editase activity 
(a)

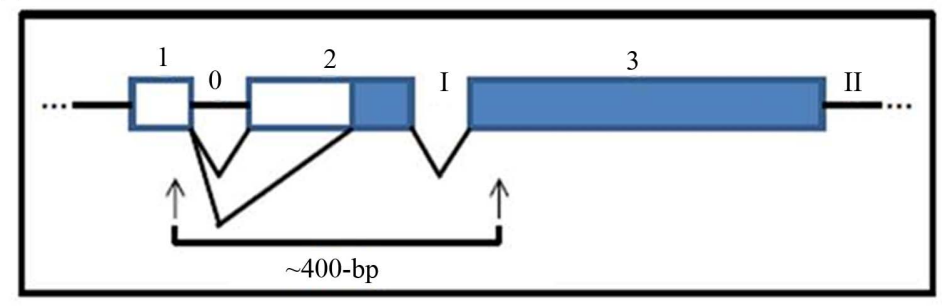

(b)

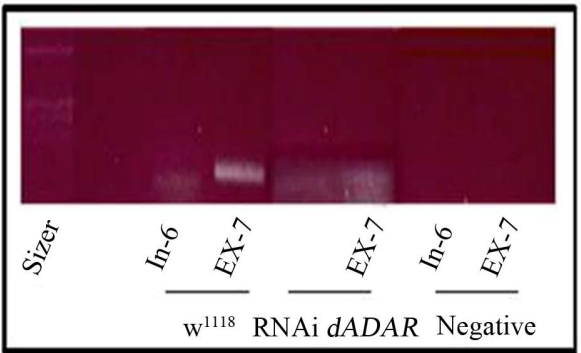

(c)

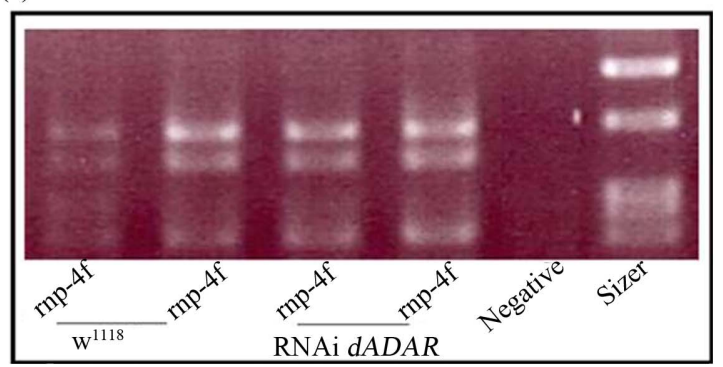

Figure 7. Influence of specific $d A D A R$ protein isoforms on 5'-UTR intron splicing regulation in rnp-4f pre-mRNA using RNAi technology. (a) Orientation diagram showing $r n p-4 f$ pre-mRNA structure around the 5'-UTR (not shaded). Exons are shown as boxes with Arabic numbers and introns as thin lines with Roman numbers. RT-PCR using primers at the indicated positions (arrows) produces three differently sized cDNA bands after gel electrophoresis: 400-, 320- and 220-bp, owing to alternative splicing, intron I being constitutively spliced [20]. (b) Verification of $d A D A R$ full-length isoform RNAi knockdown in embryos. Comparison is shown after RT-PCR between $d A D A R$ in $\mathrm{w}^{1118}$ banding pattern and relative band intensities for truncated (In-6) and full-length (Ex-7) cDNA bands and embryos in which full-length $d A D A R$ mRNA has been specifically knocked down (RNAi). Orientation diagram for $d A D A R$ RT-PCR plan is shown in Figure 4(a). (c) Comparison between rnp-4f 5'-UTR cDNA banding pattern after RT-PCR between $\mathrm{w}^{1118}$ and embryos in which full-length $d A D A R$ mRNA has been specifically knocked down (RNAi).

in vivo within Drosophila embryos [13, 32], and the possible reasons for this have previously been discussed [16]. The RNAi results reported here provide direct evidence that the full-length $D A D A R$ protein isoform is not the splicing regulator, from which it is concluded that the truncated isoform performs this role. This isoform is present in a diversity of Drosophila species, suggesting that it has been evolutionarily conserved and may therefore have a universally essential function.

\section{ACKNOWLEDGEMENTS}

We wish to thank Xiaoyun Deng from the Miami University Center for Bioinformatics and Functional Genomics for her help in running the ABI 3100 DNA sequencings. Matt Duley from the Miami University Electron Microscopy and Optical Microscopy Facility is thanked for helping with the embryo imaging studies. We also wish to thank Katherine McDowell for her help with the DIG in situ hybridization work. The Bloomington, IN Drosophila Stock Center and the Drosophila Genomics Resource Center provided valuable fly stocks and plasmids for this work. The Drosophila Species Stock Center, San Diego, CA is thanked for supplying the various species used in this work. The Developmental Studies Hybridoma Bank at The University of Iowa provided essential antibodies. We also thank the Vienna Drosophila RNAi
Center (VDRC) for transgenic RNAi stock. This work was supported by National Institutes of Health (NIH) Grant 1-R15-GM093895-01 to J. Vaughn.

\section{REFERENCES}

[1] Brand, A.H. and Perrimon, N. (1993) Targeted gene expression as a means of altering cell fates and generating dominant phenotypes. Development, 118, 401-415.

[2] Duffy, J.B. (2002) GAL4 system in Drosophila: A fly geneticist's Swiss army knife. Genesis, 34, 1-15. http://dx.doi.org/10.1002/gene. 10150

[3] Dietzl, G., Chen, D., Schnorrer, F., et al. (2007) A genome-wide transgenic RNAi library for conditional gene inactivation in Drosophila. Nature, 448, 151-157. http://dx.doi.org/10.1038/nature05954

[4] Rubin, G.M., Yandell, M.D., Wortman, J.R., et al. (2000) Comparative genomics of the eukaryotes. Science, 287, 2204-2215. http://dx.doi.org/10.1126/science.287.5461.2204

[5] Stark, A., Lin, M.F., Kheradpour, P., et al. (2007). Discovery of functional elements in 12 Drosophila genomes using evolutionary signatures. Nature, 450, 219-232. http://dx.doi.org/10.1038/nature06340 
[6] Cooper, T.A., Wan, L. and Dreyfuss, G. (2009) RNA and disease. Cell, 136, 777-793.

http://dx.doi.org/10.1016/j.cell.2009.02.011

[7] Bass, B.L. (2002) RNA editing by adenosine deaminases that act on RNA. Annual Review of Biochemistry, 71, 817-846.

http://dx.doi.org/10.1146/annurev.biochem.71.110601.13 $\underline{5501}$

[8] Nishikura, K. (2009) Functions and regulation of RNA editing by ADAR deaminases. Annual Review of Biochemistry, 79, 321-349.

http://dx.doi.org/10.1146/annurev-biochem-060208-1052 $\underline{51}$

[9] Jepson, J.E.C. and Reenan, R.A. (2008) RNA editing in regulating gene expression in the brain. Biochimica et Biophysica Acta, 1779, 459-470. http://dx.doi.org/10.1016/j.bbagrm.2007.11.009

[10] Petschek, J.P., Mermer, M.J., Scheckelhoff, M.R., Simone, A.A. and Vaughn, J.C. (1996) RNA editing in Drosophila 4f-rnp gene nuclear transcripts by multiple A-to-G conversions. Journal of Molecular Biology, 259, 885-890. http://dx.doi.org/10.1006/jmbi.1996.0365

[11] Petschek, J.P., Scheckelhoff, M.R., Mermer, M.J. and Vaughn, J.C. (1997) RNA editing and alternative splicing generate mRNA transcript diversity from the Drosophila 4f-rnp locus. Gene, 204, 267-276. http://dx.doi.org/10.1016/S0378-1119(97)00465-4

[12] Palladino, M.J., Keegan, L.P., O’Connell, M.A., and Reenan, R.A. (2000) dADAR, a Drosophila double-stranded RNA-specific adenosine deaminase is highly developmentally regulated and is itself a target for RNA editing. $R N A, 6,1004-1018$.

http://dx.doi.org/10.1017/S1355838200000248

[13] Ma, E., Tucker, M.C., Chen, Q. and Haddad, G.G. (2002) Developmental expression and enzymatic activity of pre-mRNA deaminase in Drosophila melanogaster. Molecular Brain Research, 102, 100-104. http://dx.doi.org/10.1016/S0169-328X(02)00186-9

[14] Hogg, M., Paro, S., Keegan, L.P. and O'Connell, M.A. (2011) RNA editing by mammalian ADARs. Advances in Genetics, 73, 87-119. http://dx.doi.org/10.1016/B978-0-12-380860-8.00003-3

[15] Paro, S., Li, X., O'Connell, M.A. and Keegan, L.P. (2011) Regulation and functions of ADAR in Drosophila. Current Topics in Microbiology and Immunology, 353, 221236. http://dx.doi.org/10.1007/82_2011_152

[16] Chen, J., Lakshmi, G.G., Hays, D.L., McDowell, K.M., Ma, E. and Vaughn, J.C. (2009) Spatial and temporal expression of $A A D A R$ mRNA and protein isoforms during embryogenesis in Drosophila melanogaster. Differentiation, 78, 312-320. http://dx.doi.org/10.1016/i.diff.2009.08.003

[17] Hess, K.A., Simone, A.A. and Petschek, J.P. (1996) Spatial and temporal expression of $4 f-r n p$ gene in Drosophila melanogaster. Differentiation, 61, 103-111. http://dx.doi.org/10.1046/j.1432-0436.1996.6120103.x

[18] Bell, M., Schreiner, S., Damianov, A., Reddy, R. and Bindereif, A. (2002) p110, a novel human U6 snRNP protein and U4/U6 snRNP recycling factor. The EMBO
Journal, 21, 2724-2735.

http://dx.doi.org/10.1093/emboj/21.11.2724

[19] Bae, E., Reiter, N.J., Bingman, C.A., Kwan, S.S., Lee, D., Phillips, G.N., Butcher, S.E., and Brow, D.A. (2007) Structure and interactions of the first three RNA recognition motifs of splicing factor Prp24. Journal of Molecular Biology, 367, 1447-1458. http://dx.doi.org/10.1016/j.jmb.2007.01.078

[20] Fetherson, R.A., Strock, S.B., White, K.N. and Vaughn, J.C. (2006) Alternative pre-mRNA splicing in Drosophila spliceosomal assembly factor RNP-4F during development. Gene, 371, 234-245. http://dx.doi.org/10.1016/j.gene.2005.12.025

[21] Chen, J., Concel, V.J., Bhatla, S., Rajeshwaran, R., Smith, D.L.H., Varadarajan, M., Backscheider, K.L., Bockrath, R.A., Petschek, J.P. and Vaughn, J.C. (2007) Alternative splicing of an $r n p-4 f$ mRNA isoform retaining an evolutionarily-conserved 5'-UTR intronic element is developmentally regulated and shown via RNAi to be essential for normal central nervous system development in Drosophila melanogaster. Gene, 399, 91-104. http://dx.doi.org/10.1016/j.gene.2007.04.038

[22] Lakshmi, G.G., Ghosh, S., Jones, G.P., Parikh, R., Rawlins, B.A. and Vaughn, J.C. (2012) An RNA electrophoretic mobility shift and mutational analysis of $r n p-4 f$ '-UTR intron splicing regulatory proteins in Drosophila reveals a novel new role for a $d A D A R$ protein isoform. Gene, $\mathbf{5 1 1}$, 161-168. http://dx.doi.org/10.1016/j.gene.2012.09.088

[23] Peters, N.T., Rohrbach, J.A., Zalewski, B.A., Byrkett, C.M. and Vaughn, J.C. (2003) RNA editing and regulation of Drosophila $4 f-r n p$ expression by sas-10 antisense readthrough mRNA transcripts. $R N A, \mathbf{9}, 698-710$. http://dx.doi.org/10.1261/rna.2120703

[24] Campos-Ortega, J.A. and Hartenstein, V. (1997) The embryonic development of Drosophila. Springer-Verlag, Berlin.

[25] Wolff, T. (2000) Histological techniques for the Drosophila eye. Part I: Larva and pupa. In: Sullivan, W., Ashburner, M. and Hawley, R.S., Eds., Drosophila Protocols, Cold Spring Harbor Laboratory Press, Cold Spring Harbor, New York, 201-227.

[26] Ashburner, M., Golic, K.G. and Hawley, R.S. (2005) Drosophila: A laboratory handbook, second edition. Cold Spring Harbor Laboratory Press, Cold Spring Harbor, New York.

[27] Tadros, W., Goldman, A.L., Babak, T., Menzies, F., Vardy, L., Orr-Weaver, T., Hughes, T.R., Westwood, J.T., Smibert, C.A. and Lipshitz, H.D. (2007) SMAUG is a major regulator of maternal mRNA destabilization in Drosophila and its translation is activated by the PAN GU kinase. Developmental Cell, 12, 143-155. http://dx.doi.org/10.1016/j.devcel.2006.10.005

[28] Graber, J.H., Cantor, C.R., Mohr, S.C. and Smith, T.F (1999) In silico detection of control signals: mRNA 3 '-end processing sequences in diverse species. Proceedings of the National Academy of Sciences, 96, 1405514060. http://dx.doi.org/10.1073/pnas.96.24.14055

[29] O'Connell, P. and Rosbash, M. (1984) Sequence, structure, and codon preference of the Drosophila ribosomal 
protein 49 gene. Nucleic Acids Research, 12, 5495-5513. http://dx.doi.org/10.1093/nar/12.13.5495

[30] Mignone, F., Gissi, C., Liuni, S. and Pesole, G. (2002) Untranslated regions of mRNAs. Genome Biology, 3, $1-10$.

[31] Semotok, J.L., Cooperstock, R.L., Pinder, B.D., Vari, H.K. and Lipshitz, H.D. (2005) Smaug recruits the CCR4/ POP2/NOT deadenylase complex to trigger maternal transcript localization in the early Drosophila embryo. Current Biology, 15, 284-294.

http://dx.doi.org/10.1016/j.cub.2005.01.048

[32] Hanrahan, C.J., Palladino, M.J., Ganetzky, B. and Reenan, R.A. (2000) RNA editing of the Drosophila para $\mathrm{Na}^{+}$ channel transcript: Evolutionary conservation and developmental regulation. Genetics, 155, 1149-1160. 